\title{
BMJ Open Protocol for the implementation of a stepped-care model to address fear of cancer recurrence in patients previously diagnosed with early-stage (0- II) melanoma
}

To cite: Thompson JR, Smith AL, Lo SN, et al. Protocol for the implementation of a stepped-care model to address fear of cancer recurrence in patients previously diagnosed with early-stage (0-II) melanoma. BMJ Open 2022;12:e054337. doi:10.1136/ bmjopen-2021-054337

- Prepublication history for this paper is available online. To view these files, please visit the journal online (http://dx.doi. org/10.1136/bmjopen-2021054337).

Received 15 June 2021 Accepted 04 February 2022

\section{Check for updates}

(c) Author(s) (or their employer(s)) 2022. Re-use permitted under CC BY-NC. No commercial re-use. See rights and permissions. Published by BMJ.

For numbered affiliations see end of article.

Correspondence to Jake R Thompson; jake.thompson@melanoma. org.au

\section{ABSTRACT}

Introduction Fear of cancer recurrence (FCR) is commonly reported by patients diagnosed with early-stage (0-II) melanoma and can have a significant impact on daily functioning. This study will pilot the implementation of the Melanoma Care Program, an evidence-based, psychological intervention to reduce FCR, into routine practice, using a stepped-care model.

Methods and analysis Intervention effectiveness and level of implementation will be investigated using a hybrid type I design. Between 4 weeks before and 1 week after their next dermatological appointment, patients with melanoma will be invited to complete the Fear of Cancer Recurrence Inventory Short-Form, measuring self-reported FCR severity. Using a stepped-care model, clinical cut-off points will guide the level of support offered to patients. This includes: (1) usual care, (2) Melanoma: Questions and Answers psychoeducational booklet, and (3) three or five psychotherapeutic telehealth sessions. This longitudinal, mixed-methods pilot implementation study aims to recruit 108 patients previously diagnosed with stage 0 -II melanoma. The primary effectiveness outcome is change in FCR severity over time. Secondary effectiveness outcomes include change in anxiety, depression, stress, health-related quality of life and melanoma-related knowledge over time. All outcomes are measured at baseline, within 1 week of the final telehealth session, and 6 and 12 months post-intervention. Implementation stakeholders at each study site and interested patients will provide feedback on intervention acceptability and appropriateness. Implementation stakeholders will also provide feedback on intervention cost, feasibility, fidelity and sustainability. These outcomes will be measured throughout implementation, using questionnaires and semistructured interviews/expert group discussions. Descriptive statistics, linear mixed-effects regression and thematic analysis will be used to analyse study data. Ethics and dissemination Ethics approval was granted by the Sydney Local Health District-Royal Prince Alfred Zone (2020/ETH02518), protocol number: X20-0495. Results will be disseminated through peer-reviewed
Strengths and limitations of this study

- This study aims to evaluate the pilot implementation of the evidence-based Melanoma Care Program into the routine clinical care of patients previously diagnosed with early-stage (0-II) melanoma.

- It is the first study to implement a stepped-care model to routinely screen for fear of cancer recurrence (FCR) in patients previously diagnosed with early-stage melanoma and tailor the intensity of intervention to reported FCR severity.

- Consumer representatives, practice managers, directors and clinicians have been involved throughout the study design process.

- The hybrid type I design allows for the simultaneous evaluation of clinical and implementation outcomes.

- The primary limitation of this pilot implementation study is the absence of a recruited control group; however, control group data from the Melanoma Care Program randomised controlled trial will be used as an ad hoc comparison group.

journals, conference presentations, social media and result summaries distributed to interested participants.

Trial registration details (ACTRN12621000145808).

\section{INTRODUCTION}

\section{Background and rationale}

The global incidence of melanoma is increasing, ${ }^{1}$ with an estimated 324635 individuals receiving a diagnosis of melanoma in 2020. ${ }^{2}$ Australia and New Zealand have the highest melanoma incidence rate in the world. ${ }^{3}$ In 2016, the Australian agestandardised incidence and mortality rates of melanoma were 53.5 cases per 100000 and 4.5 deaths per 100000 , respectively. ${ }^{4}$ The average 5-year survival rates of patients with 
stage I and II melanoma are $99.2 \%$ and $73.6 \%$, respectively, ${ }^{4}$ increasing the importance of their psychosocial adjustment and quality of life. Fear of cancer recurrence (FCR), defined as the fear, worry or concern that cancer may return or progress, ${ }^{5}$ is the most frequently reported challenge of this population. ${ }^{6}$ FCR is associated with: lower emotional, physical, role and social functioning, and health-related quality of life (HRQOL); poorer healthcare satisfaction; and increased reassuranceseeking behaviour, fatigue, pain, distress, anxiety and depressive symptoms. ${ }^{7}$

A meta-analysis of 23 psychological interventions targeting FCR found them to be effective; however, only one intervention, the Melanoma Care Program (MCP), focused on Australian patients with melanoma. ${ }^{8}$ The MCP is a brief, evidence-based psychological intervention developed to address FCR in patients with a previous diagnosis of early-stage melanoma at risk of developing new primary disease. ${ }^{9} 10$ The intervention consists of two components: (1) a Melanoma: Questions and Answers (MQA) psychoeducational booklet, ${ }^{10}{ }^{11}$ and (2) three psychotherapeutic telehealth sessions scheduled around patients' dermatological visits. ${ }^{10}$ This is the first intervention specifically developed for patients with a previous diagnosis of early-stage melanoma. When investigated in a randomised controlled trial, intervention participants reported significantly lower FCR severity compared with a control group immediately post-intervention and at 6 -month follow-up, ${ }^{9}$ with effects sustained at 12-month follow-up. ${ }^{12}$ The intervention was also well accepted by patients $^{9}$ and cost-effective. ${ }^{13}$ While the efficacy of this intervention was established, the randomised controlled trial did not assess patients' FCR severity prior to trial enrolment to tailor intervention intensity to patient need. The present protocol outlines a pilot implementation study to translate this evidence-based intervention into real-world clinical settings, using a stepped-care approach. Patients with a previous diagnosis of early-stage melanoma attending routine dermatological appointments will be screened for FCR, allowing the intensity of support to match the severity of the patients' FCR.

\section{Study aims and hypotheses}

The primary aim of this study is to examine the effectiveness of a stepped-care model offering the MCP (henceforth referred to as the 'Melanoma Care Programstepped-care intervention' or MCP-SCI) in reducing FCR severity in patients with a previous diagnosis of early-stage melanoma who are identified as having elevated FCR in routine clinical practice.

Secondary aims include:

- Evaluation of the effects of the MCP-SCI on patientreported depression, anxiety, stress, melanomarelated knowledge, HRQOL and further aspects of FCR: triggers, psychological distress, coping strategies, functional impairments, insight and reassurance.

- Evaluation of the sustainability of routine implementation of the MCP-SCI in real-world clinical settings by documenting barriers (eg, low screening uptake, time and cost of screening) and facilitators (eg, participant engagement and screening adherence) and assessing the usefulness of strategies to address barriers.

It is hypothesised that:

1. Patients who report elevated FCR and receive the MCP-SCI will report immediately, and at 6-month and 12-month follow-up:

- A significant reduction in FCR severity.

- A decrease in FCR-related triggers, psychological distress, functional impairments, reassuranceseeking behaviour and patient-reported levels of depression, anxiety and stress.

- An increase in FCR-related coping strategies and insight, melanoma-related knowledge and HRQOL compared with baseline scores.

2. The implementation of the MCP-SCI will be considered:

- Acceptable and appropriate by patients who receive the intervention.

- Acceptable, appropriate, feasible and sustainable by implementation stakeholders.

3. The MCP-SCI will be delivered with high fidelity and adherence to the therapist manual.

\section{METHODS}

\section{Study design}

Translational research investigates the degree to which an evidence-based practice retains its effectiveness when implemented into 'real-world' settings. ${ }^{14}$ The hybrid effectiveness-implementation design, which focuses on assessing both the effectiveness and implementation of an evidence-based practice, is commonly used in translational research. ${ }^{15}$ Three variations of this design exist, based on the relative focus that is placed a priori on effectiveness and implementation outcomes. Type I designs primarily evaluate the health and well-being impact of an evidence-based practice in real-world settings, while also gathering contextual information on the implementation process to guide future implementation efforts. ${ }^{15}$ Thus, a type I design was selected for this study to investigate the effects of the MCP-SCI in routine practice, while evidence concerning its long-term sustainability is gathered to guide more extensive implementation efforts in the future.

\section{Setting}

Implementation will take place at two of the three dermatology clinics specialising in the diagnosis and treatment of melanoma that participated in the MCP randomised controlled trial. ${ }^{910}$ The first study site, Melanoma Dermatology, is located within the Poche Centre at Melanoma Institute Australia (MIA), the world's largest melanoma research and treatment facility. The second study site, Sydney Melanoma Diagnostic Centre, is also associated with MIA and is located at Royal Prince Alfred Hospital. Both study sites are located in metropolitan Sydney, 
Table 1 Participant inclusion and exclusion criteria

\begin{tabular}{|c|c|c|}
\hline & Inclusion criteria & Exclusion criteria \\
\hline Patients with melanoma & $\begin{array}{l}\text { Current or previous diagnosis of stage } 0, \text { I or II } \\
\text { melanoma and currently completing follow-up at } \\
\text { one of the study sites. } \\
\text { Sufficient English language skills and cognitive } \\
\text { ability to understand study materials and provide } \\
\text { informed consent. } \\
\text { Sufficient hearing to participate in telehealth } \\
\text { consultations. } \\
\text { Aged } 18 \text { years or older. }\end{array}$ & $\begin{array}{l}\text { Current or previous diagnosis of stage III or IV } \\
\text { melanoma, irrespective of current disease status. } \\
\text { At high risk of, but no previous diagnosis of } \\
\text { melanoma. } \\
\text { Significant cognitive impairment that would } \\
\text { prevent understanding of the study materials and } \\
\text { ability to provide informed consent. } \\
\text { Significant hearing impairment preventing } \\
\text { participation in telehealth consultations. } \\
\text { Current diagnosis of severe depression, } \\
\text { psychotic illness or other serious psychiatric } \\
\text { condition. } \\
\text { Below } 18 \text { years of age. }\end{array}$ \\
\hline $\begin{array}{l}\text { Implementation } \\
\text { stakeholders }\end{array}$ & $\begin{array}{l}\text { Member of the MCP randomised controlled trial } \\
\text { investigative team, } \\
\text { OR } \\
\text { Current employee of Melanoma Institute } \\
\text { Australia or Sydney Melanoma Diagnostic } \\
\text { Centre and directly involved in the } \\
\text { implementation of the intervention. } \\
\text { Sufficient English language skills and cognitive } \\
\text { ability to understand study materials and provide } \\
\text { informed consent. } \\
\text { Aged } 18 \text { years or older. }\end{array}$ & $\begin{array}{l}\text { Significant cognitive impairment that would } \\
\text { prevent understanding of the study materials and } \\
\text { ability to provide informed consent. } \\
\text { Employed by Melanoma Institute Australia } \\
\text { or Sydney Melanoma Diagnostic Centre but } \\
\text { not directly involved in implementation of the } \\
\text { stepped-care intervention. } \\
\text { Below } 18 \text { years of age. }\end{array}$ \\
\hline
\end{tabular}

MCP, Melanoma Care Program.

Australia; are mixed public and private practices that have extensive experience in conducting melanomarelated research and implementation studies; have strong organisational emphasis on multidisciplinary collaboration, research and clinician training; consist of roughly a dozen clinicians and administration staff; and primarily see patients with melanoma at high risk of recurrence.

\section{Participant selection}

Two groups of participants will be included: (1) patients with a current or previous diagnosis of early-stage melanoma who have an upcoming follow-up appointment at either of the study sites, and (2) implementation stakeholders, including investigators of the MCP randomised controlled trial, and individuals who are involved in the implementation of the MCP-SCI at one of the study sites (ie, dermatologists, nurses, practice managers, administration staff). Table 1 outlines the study inclusion and exclusion criteria.

\section{Participant recruitment}

Patients

Four weeks prior to their routine scheduled appointment, patients will be invited to participate via an automated text message. This time frame allows for individuals with high FCR to be identified and offered the intervention prior to the week of their appointment, when anxiety is likely to be greatest. ${ }^{16}$ The text message invitation contains a brief introduction to the study and a link to MIA's Research Electronic Data Capture webpage, which includes a landing page describing the study, participant information statement, consent form, Fear of Cancer Recurrence Inventory Short Form (FCRI-SF $)^{17}$ and relevant questionnaires. During an eligible patient's appointment, their clinician will check that the text message was received and answer any questions about the study. If the patient did not receive the text message, the clinician can discuss the study with the patient and collect their contact details if interested. A research assistant will then call the patient within 48 hours of the appointment to discuss the study.

\section{Implementation stakeholders}

The chief investigator at each study site will approach potential implementation stakeholders via email or in person. Additionally, members of the investigative team of the MCP randomised controlled trial will be invited to participate as implementation stakeholders, as these individuals have first-hand experience with the intervention and may foresee possible implementation issues. A reminder invitation will be sent 2 weeks following initial contact if no response is received.

The recruitment of implementation stakeholders began in June 2021. Patient recruitment began in January 2022. Study completion is expected by October 2023.

\section{Intervention description}

MQA booklet and psychotherapeutic telehealth sessions

The MCP intervention consists of the MQA booklet and psychotherapeutic telehealth sessions. These two components have not been substantially altered for this pilot implementation study. Table 2 provides a brief 
Table 2 Description of the MCP intervention

$M C P$ randomised controlled trial ${ }^{10}$

\begin{tabular}{ll}
\hline Study sites & $\begin{array}{l}\text { 1. Sydney Melanoma Diagnostic Centre } \\
\text { 2. Poche Centre, MIA }\end{array}$ \\
3. Newcastle Skin Check Clinic \\
Screening & N/A \\
$\begin{array}{l}\text { Melanoma: Questions and } \\
\text { Answers (MQA) booklet }\end{array}$ & $\begin{array}{l}\text { A purpose-designed, psychoeducational booklet } \\
\text { developed by a multidisciplinary team and } \\
\text { published in March } 2014 \text { featuring comprehensive } \\
\text { information on a range of topics identified } \\
\text { as important to patients with melanoma }\end{array}$ \\
& $\begin{array}{l}\text { melanoma diagnosis, treatment, recurrence rates, } \\
\text { prevention and strategies to address and cope } \\
\text { with FCR. Patients have found this booklet both } \\
\text { satisfactory and beneficial, with responses being } \\
\text { overwhelmingly positive. }\end{array}$
\end{tabular}

Psychotherapeutic telehealth sessions
Three telephone-based sessions with a trained psychologist based on the principles of brief, psychodynamically oriented psychotherapy, aiming to provide patients with melanoma effective emotional and behavioural coping strategies. These sessions were guided by a psychologist manual, outlining the different features and discussion topics of the first, middle and final sessions.

\section{Pilot implementation study}

1. Sydney Melanoma Diagnostic Centre

2. Poche Centre, MIA

\section{Conducted using the FCRI-SF.}

The MQA booklet's design and information was updated and made complimentary to MIA's Your Guide to Early Melanoma (3rd Edition) booklet, ${ }^{45}$ which is offered to patients with early-stage melanoma as a part of standard care at MIA. As both booklets contain similar information on melanoma diagnosis and treatments, information in the MQA booklet on these topics was summarised to reduce patient burden. This review was completed by a consumer representative, melanoma clinicians and researchers to ensure it contains up-to-date information as of publishing in December 2021. All patients who participate in this study will be offered a copy of MIA's booklet as a part of their standard care, with patients who receive the intervention also offered a copy of the MQA booklet.

Based on the results of the MCP randomised controlled trial and discussion with its lead investigators, patients with significantly elevated FCR at baseline would have likely benefited from more than three telehealth sessions. ${ }^{12}$ Thus, this study will offer these patients a total of five sessions rather than three. It is important to note that although a patient will be offered a number of telehealth sessions, the number of sessions received will be dependent on the clinical opinion of the psychologist(s) and patient need. The psychologist manual has not been altered for this study (patients offered five sessions will receive one 'first', three 'middle' and one 'final' session) and these sessions will take place via telephone or video-conferencing software.

\begin{tabular}{|c|c|}
\hline $\begin{array}{l}\text { Timing of telehealth } \\
\text { sessions }\end{array}$ & $\begin{array}{l}\text { The first session was held } 1 \text { week before the } \\
\text { upcoming dermatology appointment of the patient } \\
\text { with melanoma. All subsequent sessions were held } \\
\text { on a fortnightly basis. }\end{array}$ \\
\hline Primary study outcome & $\begin{array}{l}\text { Change in FCR severity over time measured using } \\
\text { the FCRI-SF. }\end{array}$ \\
\hline $\begin{array}{l}\text { Primary outcome collection } \\
\text { timeline }\end{array}$ & $\begin{array}{l}\text { Baseline: 4-6 weeks before upcoming } \\
\text { dermatology appointment } \\
\text { Follow-up 1: } 1 \text { week after final telehealth session } \\
\text { Follow-up 2: } 6 \text { months after final telehealth } \\
\text { session } \\
\text { Follow-up 3: } 12 \text { months after final telehealth } \\
\text { session }\end{array}$ \\
\hline
\end{tabular}

The first session will be held as soon as possible after FCR screening, which can take place between 4 weeks before to 1 week after the upcoming dermatology appointment of the patient with melanoma. All subsequent sessions will be held on a fortnightly basis.

Change in FCR severity over time measured using the FCRISF.

Baseline: ranging from 4 weeks before to 1 week after upcoming dermatology appointment

Follow-up 1: 1 week after final telehealth session

- Follow-up 2: 6 months after final telehealth session

- Follow-up 3: 12 months after final telehealth session

FCR, fear of cancer recurrence; FCRI-SF, Fear of Cancer Recurrence Inventory 9-item Short Form; MCP, Melanoma Care Program; MIA, Melanoma Institute Australia; N/A, Not applicable.

description of the MCP intervention provided to patients in the randomised controlled trial and outlines the justification for any modifications made for the present implementation study.

The content of the psychotherapeutic telehealth sessions is provided in table 3 .

\section{Stepped-care model of intervention delivery}

The addition of a stepped-care model of care will allow the intervention to be tailored to each patient's severity of FCR, potentially maximising overall benefit and service provision efficiency while conserving resources. ${ }^{18}$
Patients will be invited to complete FCR screening using the FCRI-SF. The FCRI-SF is measured using a nine-item, 5-point Likert scale with scores ranging from 0 to 36, with higher scores indicating greater FCR severity. ${ }^{17}$ Multiple cut-off scores have been suggested in the literature. For the purpose of this pilot implementation study, a cut-off score of $\geq 13^{19}$ will be used to identify patients with clinically indicative levels of FCR to receive the MCP-SCI, with a second cut-off core of $\geq 22^{20}$ used to identify patients with more severe levels of FCR at baseline (table 4). These cut-off scores were chosen as a preference was placed on sensitivity over specificity to ensure patients experiencing 
Table 3 Outline of psychotherapeutic telehealth sessions

\begin{tabular}{ll}
\hline Session & Content $^{10}$ \\
\hline Introduction & $\begin{array}{l}\text { The psychologist introduces themselves to } \\
\text { the patient, checks all materials have been } \\
\text { received, reconfirms consent and schedules } \\
\text { the first session. }\end{array}$ \\
Session 1 & $\begin{array}{l}\text { The psychologist assesses patient needs, } \\
\text { referring to the MQA booklet where } \\
\text { appropriate when discussing any concerns } \\
\text { or unmet needs the patient has. }\end{array}$ \\
Sessions 2-4 & $\begin{array}{l}\text { The psychologist reviews previous session(s) } \\
\text { with the patient and discusses any difficulties } \\
\text { that have arisen since. The psychologist } \\
\text { will continue to address the unmet needs } \\
\text { of patients using the MQA booklet where } \\
\text { possible. }\end{array}$ \\
$\begin{array}{l}\text { The psychologist reviews all previous } \\
\text { sessions and addresses any new difficulties. }\end{array}$ \\
$\begin{array}{l}\text { The psychologist discusses the degree } \\
\text { to which patient unmet needs have been } \\
\text { addressed, new strategies to address } \\
\text { possible future concerns and referral for } \\
\text { further support if required. }\end{array}$ \\
\end{tabular}

MQA, Melanoma: Questions and Answers.

FCR are captured, and the MCP randomised controlled trial subgroup analysis finding participants scoring $\geq 13$ at baseline experienced a significant decrease in FCR severity at 6-month follow-up, ${ }^{9}$ whereas participants scoring $\geq 22$ at baseline experienced no significant decrease, ${ }^{12}$ which investigators attributed to potential dose-response effect. Hence, it was decided to offer two additional sessions to those who score $\geq 22$ at baseline to investigate its effects.

Patients triaged to step 1 (no/low FCR) will receive usual care, consisting of clinical follow-up and MIA's Your Guide to Early Melanoma (Third Edition) booklet. Patients triaged to step 2 (moderate FCR) and step 3 (severe FCR) will continue to receive usual care as well as being offered the MCP intervention, with the difference between these steps being the number of telehealth sessions offered to the patient (table 3). The psychologist(s) will contact patients to schedule their first session, ideally conducting the first session before the patients' upcoming appointment. Subsequent telehealth sessions will be conducted on a flexible 2-week basis.

\section{Data collection}

Patients

In addition to completing the FCRI-SF, patients triaged to step 1 (no/low FCR) will complete a demographic questionnaire. Patients triaged to step 2 (moderate FCR) and step 3 (severe FCR) will complete the demographic questionnaire plus a baseline questionnaire collecting data relating to outcome measures of interest. Patients triaged to step 2 or 3 will also complete questionnaires within 1 week of completing their final telehealth session and at 6-month and 12-month follow-up. All patients who receive the intervention will be invited to participate in a semistructured interview during their first follow-up questionnaire to explore their experiences of the MCPSCI. Recruitment will continue until thematic saturation is reached, and purposeful sampling used to ensure a range of experiences are captured. The Theoretical Framework of Acceptability ${ }^{21}$ will be used to guide these semistructured interviews. This framework consists of seven constructs relevant to intervention acceptability: affective attitude, burden, perceived effectiveness, ethicality, intervention coherence, opportunity costs and self-efficacy.

Patients will choose whether to complete questionnaires electronically or in paper format. A reminder email/letter will be sent to patients who do not provide a response after 2 weeks, with a telephone reminder after 4 weeks.

Table 4 Stepped-care model

\begin{tabular}{|c|c|c|c|c|}
\hline Steps of intervention & $\begin{array}{l}\text { FCRI-SF clinical cut- } \\
\text { off score }\end{array}$ & Usual care* & $\begin{array}{l}\text { MQA } \\
\text { booklet }\end{array}$ & $\begin{array}{l}\text { Number of offered } \\
\text { psychotherapeutic } \\
\text { telehealth sessions }\end{array}$ \\
\hline \multicolumn{5}{|l|}{ Step 1} \\
\hline No/low FCR & $<13$ & $\checkmark$ & - & 0 \\
\hline \multicolumn{5}{|l|}{ Step 2} \\
\hline Moderate FCR & $13-21$ & $\checkmark$ & $\checkmark$ & 3 \\
\hline Severe FCR & $\geq 22$ & $\checkmark$ & $\checkmark$ & 5 \\
\hline \multicolumn{5}{|l|}{ Step 4} \\
\hline $\begin{array}{l}\text { Significant comorbid mental } \\
\text { health condition }\end{array}$ & N/A† & $\checkmark$ & $\checkmark$ & Referralł \\
\hline
\end{tabular}

*Patient education and support as per usual clinical practice, including the provision of MIA's Your Guide to Early Melanoma (Third Edition) booklet. †ldentified through baseline questionnaire and clinical judgement during telehealth sessions.

$\ddagger$ Referred to community mental health specialist or general practitioner.

FCR, fear of cancer recurrence; FCRI-SF, Fear of Cancer Recurrence Inventory 9-item Short Form; MIA, Melanoma Institute Australia; MQA,

Melanoma: Questions and Answers; N/A, Not applicable. 
Implementation stakeholders

Three expert groups will be formed to explore the perceptions of implementation stakeholders to gather information about barriers and facilitators to implementation. The first group, consisting of investigators of the MCP randomised controlled trial, will meet preimplementation to discuss barriers and facilitators experienced during the trial and any foreseeable barriers during implementation in routine clinical practice. The second and third groups, consisting of implementation stakeholders at the two study sites, will meet 3 months prior to, and quarterly throughout implementation, to discuss key barriers and strategies to address them, meeting a final time 3 months post-implementation to discuss long-term sustainability of the intervention. These expert group discussions will be guided by the Consolidated Framework for Implementation Research and will be audio-recorded and transcribed for thematic analysis. ${ }^{22}$

Formative evaluation will be used to assess the effectiveness of any strategies put in place to address barriers that are identified during the implementation process, ${ }^{14}$ with information collected shared with investigators and stakeholders allowing the implementation process to adapt to any barriers. Summaries of each expert group and agreed upon modifications will be provided to the study sites within a week of each expert group discussion, allowing implementation stakeholders to enact any changes. This will allow investigators to evaluate the effects of strategies used to address barriers to implementation.

At the conclusion of each expert group, implementation stakeholders will be offered questionnaires to quantitatively explore the acceptability, appropriateness and feasibility of the intervention.

\section{Outcomes}

Consistent with the hybrid type I design, the primary outcome is the effectiveness of the MCP-SCI in reducing patient FCR severity. A summary of the outcome assessment methods is presented in table 5 .

\section{Primary outcome}

The primary outcome of this study is change in patient self-reported levels of FCR severity over time, measured using the FCRI-SF, the severity subscale of the Fear of Cancer Recurrence Inventory 42-item Form (FCRI). ${ }^{17}$

\section{Secondary effectiveness outcomes}

Changes over time in all other subscales of the FCRI (triggers, psychological distress, coping strategies, functional impairments, insight and reassurance) will be measured and reported as secondary outcomes. The FCRI consists of 42 items that patients answer using a 5-point Likert scale. Higher scores indicate higher levels of FCR. The FCRI has demonstrated psychometric properties (table 5) and has been validated in Australians with a history of earlystage melanoma. ${ }^{23}$

Change over time in melanoma-related knowledge will be measured using a purpose-designed questionnaire, adapted from the MCP randomised controlled trial. This questionnaire was updated in tandem with the MQA booklet, to ensure the questions and answers continue to reflect the information provided in the booklet. Higher scores on this scale correspond to higher levels of melanoma-related knowledge, which is measured using multiple choice, true/false and yes/no style questions.

Changes over time in depression, anxiety and stress will be measured using the Depression, Anxiety and Stress Scales 21-item Short Form (DASS-21). ${ }^{24}$ The DASS-21 is measured using a 4-point Likert scale, with higher scores indicating more severe symptoms of depression, anxiety or stress. The DASS-21 has demonstrated psychometric properties (see table 5) and has clinical cut-off ${ }^{24}$ and clinically meaningful ${ }^{25}$ scores defined.

Change over time in $H R Q O L$ will be measured using the Assessment of Quality of Life-8 Dimensions (AQOL8D). ${ }^{26}$ The AQOL-8D contains 35 questions to which patients respond using Likert scales ranging from 4 to 6 points. The AQOL-8D has demonstrated psychometric properties (see table 5). The AQOL-8D scores comprise two super dimensions (physical and psychosocial) consisting of eight smaller dimensions (independent living, pain, senses, mental health, happiness, coping, relationships and self-worth). Higher scores indicate worse quality of life.

\section{Secondary implementation outcomes}

The acceptability and appropriateness of the MCP-SCI from a patient's perspective will be quantitatively measured using the Acceptability of Intervention Measure and Intervention Appropriateness Measure, respectively. ${ }^{27}$ Each of these measures consists of four positively worded items, measured on a 5-point Likert scale. As no cut-off scores exist, scores of $4 / 5$ (agree) and 5/5 (strongly agree) will be used to indicate that the MCP-SCI is considered acceptable and appropriate by patients. Furthermore, semistructured interviews with patients will be used to further explore the perceptions of patients. Acceptability will also be measured using intervention adherence rates.

Implementation stakeholders will provide further feedback regarding the acceptability and appropriateness of the MCP-SCI. This will be quantitatively measured using the Acceptability of Intervention and Intervention Appropriateness Measures. Furthermore, implementation stakeholders will also provide feedback regarding the feasibility or the MCP-SCI, quantitatively measured using the Feasibility of Intervention Measure. ${ }^{27}$ Lastly, expert group discussions with implementation stakeholders will be used to further explore their perceptions of the MCP-SCI.

The cost of implementation will be reported using process data, which will include costs associated with the MQA booklet (ie, time to update, graphic design and printing), training, salary of a psychologist, text messaging, online screening and survey development, stationery, transcribing interviews and any other incidental expenses. These expenditures will be categorised 


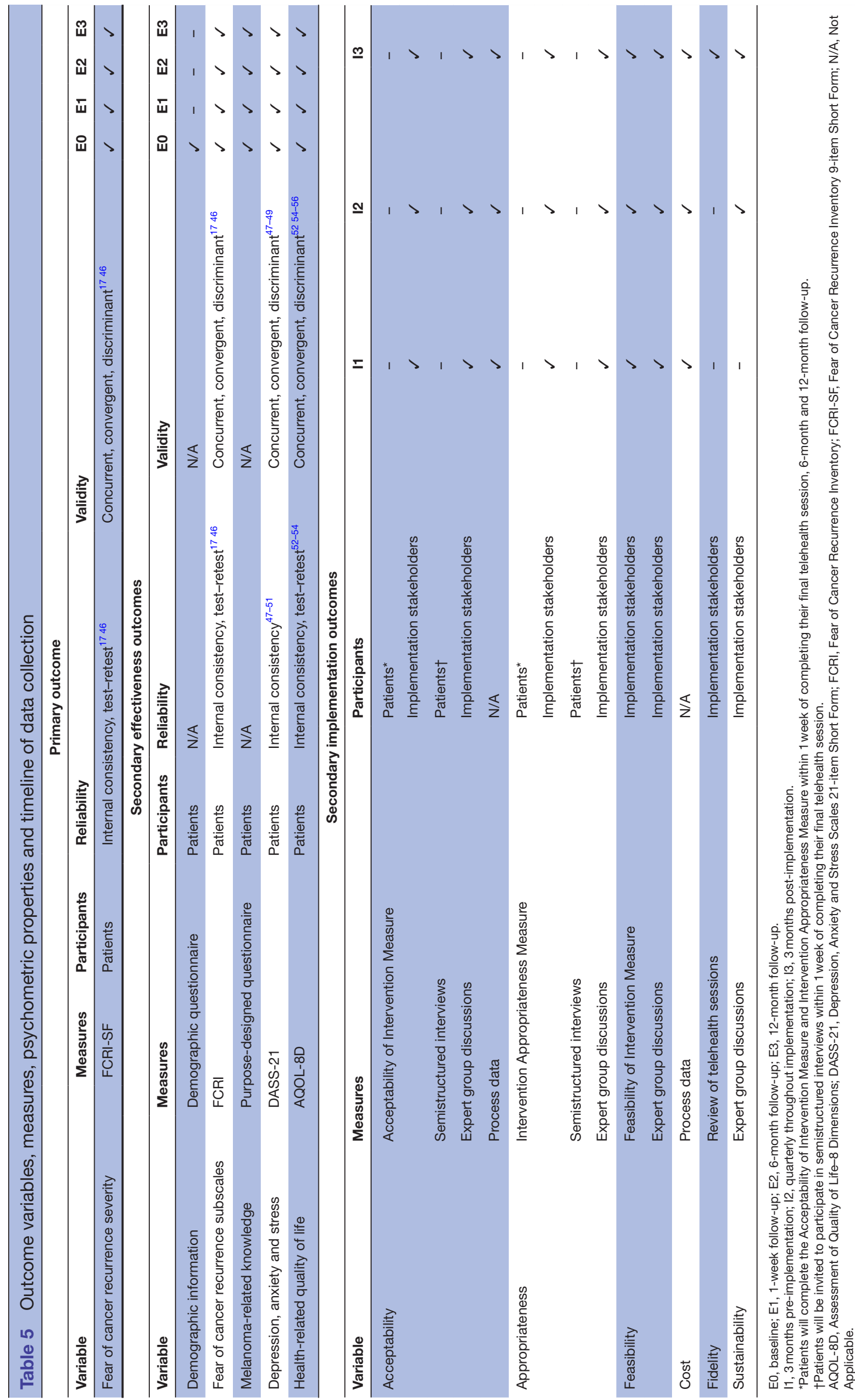

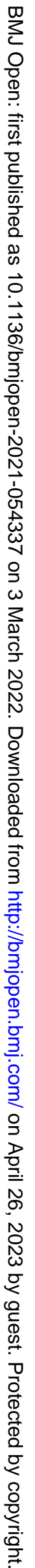


into costs associated with research, initiating implementation and ongoing implementation.

Fidelity of the telehealth sessions to the psychologist manual will be assessed using a purpose-designed fidelity checklist adapted from the MCP. ${ }^{9}$ This checklist includes items specifically designed to review the content of each session, including the items from the Comparative Psychotherapy Process Scale,${ }^{28}$ Revised Cognitive Therapy Scale, ${ }^{29}$ and Interpretive and Supportive Technique Scale. ${ }^{30}$ To ensure the psychologist manual is adequately followed, $10 \%$ of conducted telehealth sessions will be randomly reviewed and assessed.

Finally, sustainability will be assessed through the degree to which the intervention has been incorporated into routine clinical care at the study sites. The sustainability of the MCP-SCI will be discussed with implementation stakeholders through expert group discussions.

\section{Sample size}

At 12 months post-intervention, the MCP randomised controlled trial demonstrated a reduction in FCR severity of $-1.41 .{ }^{12}$ Based on this value, a sample size of 86 will provide $90 \%$ power to detect an overall before/after difference of -1.41 in FCR severity between baseline and 12 months post-intervention. This sample size calculation is based on a paired mean difference design with an SD of 4.0 and type 1 (alpha) error rate set to $0.05 .^{31}$ Assuming a conservative lost-to-follow-up rate of $20 \%$, a final sample of 108 patients across both study sites will be recruited and offered the MCP-SCI. As it is anticipated that approximately $63 \%$ of patients who complete screening will be offered the intervention, ${ }^{32}$ an estimated 172 patients will complete screening, with recruitment continuing until the required sample of 108 patients is achieved.

\section{Data analysis plan}

All patients who receive the intervention will be analysed as one group with the number of psychotherapeutic telehealth sessions received and baseline FCR scores treated as covariates. Linear mixed-effects regression will be used to analyse the effect of the intervention on patient psychosocial outcomes, as it can robustly deal with missing data and perform hypothesis testing on longitudinal data. ${ }^{33}$ Furthermore, the FCR trajectory of both the intervention group and the control group recruited in the MCP randomised controlled trial ${ }^{12}$ will be graphically displayed in the same figure using mean scores at baseline, 1-week, 6-month and 12-month follow-up with $95 \%$ CIs for ad hoc comparison. No formal statistical inference will be performed to compare the two groups. Moderation analysis will also be used to examine the effects of covariates on the relationship between all outcomes and independent variables through linear regression. ${ }^{34}$ Thematic analysis will be used to analyse the semistructured interviews and expert group discussions conducted throughout the study for common themes regarding facilitators and barriers. ${ }^{35}$ Quantitative analysis will be completed in IBM SPSS Statistics V.26 (IBM Corp) and
RStudio (RStudio Team 2019, V.1.2.5033); qualitative analysis will be conducted using NVivo V.12 Plus (QSR International).

\section{Ethics}

Ethical approval was received from the Sydney Local Health District-Royal Prince Alfred Zone (2020/ ETH02518). Any future amendments to the protocol will be approved by the Steering Committee, Human Research Ethics Council, site approval boards and the corresponding clinical trial registry updated. Participants enrolled in the study will be informed by JRT. Based on the MCP randomised controlled trial, it is unlikely that patient participants will experience adverse effects from the stepped-care intervention, as only three participants (4\%) found discussing their melanoma experiences with a psychologist confronting. ${ }^{36}$ Any discomfort will be addressed during the telehealth sessions. Furthermore, any participants identified to have a significant comorbid mental health condition will be referred for community mental health support to better address their needs.

\section{Dissemination plans}

Results will be shared with academics, researchers, clinicians, interested patients and other key stakeholders. Results will be disseminated to peer-reviewed journals, scientific meetings and conferences and reported according to the Standards for Reporting Implementation Studies statement. ${ }^{37}$ The associated checklist ${ }^{38}$ will be used to ensure all relevant aspects of the intervention study are included in analysis and reporting. Authorship will be determined by the criteria outlined in the International Committee for Medical Journal Editors. ${ }^{39}$

\section{Data availability}

To facilitate research transparency, reproducibility and accuracy, de-identified data will be available for sharing. Interested researchers can contact the corresponding investigator following the publication of the 12-month follow-up data. Data access will be granted to the projects that are considered by the investigative team to be methodologically sound and Human Research Ethics Committee approved. The investigative team will create a project-specific workspace within MIA's secure server, which will house the de-identified data and technical appendices.

\section{Patient and public involvement}

The design of this study, its aim and outcome measures are a result of the positive outcomes and satisfaction reported by the participants of the MCP randomised controlled trial, ${ }^{36}$ as well as the clinical experiences of the investigative team regarding patients experiencing anxiety and FCR. The investigative team also includes a consumer representative who has provided guidance on the design of the study and its materials, approved of the intervention's possible burden on patients and will continue to provide guidance throughout implementation as barriers are identified. No consumers are directly 
involved in the recruitment process. A lay summary of results will be provided to any interested patients and posted on the MIA website.

\section{DISCUSSION \\ Strengths}

This pilot implementation study represents the next logical step in the translation of an evidence-based psychotherapeutic intervention to reduce FCR in patients with a previous diagnosis of early-stage melanoma into routine clinical practice. The study design will allow for concurrent assessment of effectiveness and implementation variables using a mixed-methods design which includes quantitative data obtained through use of validated and accepted outcome measures, with contextual information obtained from interviews and expert groups. Screening will be used to identify patients experiencing elevated FCR and ensure patients are offered the appropriate level of support to address their needs. This screening will take place between 4 weeks before and 1 week after a scheduled appointment in an attempt to capture the background levels of FCR experienced by the patient, as fear often increases in the week before an appointment. ${ }^{16}$ Consumer representatives, practice managers, directors and clinicians were included in the study design process, ensuring the intervention has the utmost relevance to patient needs and will suit the organisational structure of the study sites.

\section{Limitations}

Similar to the MCP, the study design precludes determination of the relative contribution of the MQA booklet and psychotherapeutic telehealth sessions in achieving outcomes. Further studies may be designed to systematically investigate this. Furthermore, a control group will not be recruited, as withholding evidence-based intervention from patients who may screen high on FCR in the control group was not considered ethical. Available control group data from the MCP randomised controlled trial will act as a comparison group to estimate 12-month FCR trajectories without an intervention. Furthermore, there is a risk of recruitment bias as patients with severe FCR may avoid participating in this study. The extent of this recruitment bias will be estimated by comparing recruitment rates with other interventional studies in the melanoma literature.

\section{Significance}

Information on the implementation of evidence-based psychosocial interventions into routine melanoma practice is sparse. Only one study was identified that evaluated the implementation of an FCR intervention into routine practice. The Fear-Less ${ }^{40}$ Study evaluated a stepped-care model on patients with metastatic (stage IV) melanoma, using the ConquerFear ${ }^{41}$ intervention, which, in a clinical trial, was found to be effective in reducing FCR in patients with breast and colorectal cancer and melanoma. ${ }^{42}$
Fear-Less was found to be both acceptable and feasible. The small sample size precluded determination whether the observed reduction in FCR was statistically significant or clinically meaningful. This study will be the first to provide a stepped-care intervention for patients with a previous diagnosis of early-stage melanoma reporting elevated FCR in routine clinical practice, using an intervention that has been specifically created for patients with melanoma; addresses both the international ${ }^{43}$ and Australian $^{44}$ research agenda for FCR, specifically as it uses a stepped-care model, facilitates routine implementation of an evidence-based intervention, and provides access to telehealth interventions to patients outside of clinical trials; is sufficiently powered to assess the impact of the intervention on FCR severity; and will be the first to investigate the acceptability, appropriateness, feasibility, fidelity and sustainability of a psychosocial intervention implemented into routine practice to address FCR in patients previously diagnosed with early-stage melanoma, from both the consumer and service-provider perspective. The implementation information obtained may be used in future implementation efforts as research moves from the strict confines of clinical trials into real-world settings.

\section{Author affiliations}

${ }^{1}$ Melanoma Institute Australia, University of Sydney, North Sydney, New South Wales, Australia

${ }^{2}$ Australian Institute of Health Innovation, Macquarie University, Sydney, New South Wales, Australia

${ }^{3}$ The Daffodil Centre, The University of Sydney, a joint venture with Cancer Council NSW, Sydney, New South Wales, Australia

${ }^{4}$ Cincinnati Children's Center for Heart Disease and Mental Health, Cincinnati Children's Hospital Medical Center, Cincinnati, Ohio, USA

${ }^{5}$ Department of Pediatrics, University of Cincinnati College of Medicine, Cincinnati, Ohio, USA

${ }^{6}$ Department of Melanoma and Surgical Oncology, Royal Prince Alfred Hospital, Camperdown, New South Wales, Australia

${ }^{7}$ NHMRC Clinical Trials Centre, The University of Sydney, Sydney, New South Wales, Australia

${ }^{8}$ Consumer Representative, Sydney, New South Wales, Australia

${ }^{9}$ Faculty of Medicine and Health, University of New South Wales, Kensington, New South Wales, Australia

${ }^{10}$ Sydney Melanoma Diagnostic Centre, Royal Prince Alfred Hospital, Sydney, New South Wales, Australia

${ }^{11}$ Melanoma and Skin Service, Peter MacCallum Cancer Centre, Melbourne, Victoria, Australia

${ }^{12}$ Westmead Institute for Medical Research, The University of Sydney, Sydney, New South Wales, Australia

Acknowledgements The authors wish to thank Ms Ivy Tan for software assistance, Dr Niamh 0'Sullivan for assisting with the Melanoma: Questions and Answers booklet review, Melanoma Institute Australia and Sydney Melanoma Diagnostic Centre.

Contributors NAK, RS, MD, JRT, IB and AEC were responsible for the concept of this study. A Steering Committee comprising of JRT, IB, ALS, LS, LM, PG, NAK, RS, $\mathrm{MD}, \mathrm{HS}, \mathrm{AEC}, \mathrm{SL}$ and DM contributed to study design, intervention and material development. JRT, IB, LM, PG and ALS will be responsible for overseeing the implementation process. JRT, IB and ALS will be responsible for data collection and management. JRT, IB, ALS, MD and SL will conduct the statistical analysis of results. All authors will be involved in results interpretation. All authors contributed and approved this manuscript.

Funding This implementation trial was supported by the Melanoma Centre of Research Excellence (The University of Sydney, New South Wales, Australia), which 
is funded by the National Health and Medical Research Council of Australia (grant number: 1135285). This work was also supported by Melanoma Institute Australia and the Bill and Patricia Ritchie Foundation. NAK is the recipient of a National Heart Foundation of Australia Future Leader Fellowship (101229) and support from the Heart Institute Research Core at Cincinnati Children's Hospital. AEC has received a National Health and Medical Research Council Career Development Fellowship (1147843).

Disclaimer The funding sources had no role in the design of this study and will not have any role during its implementation, analysis or decision to publish results.

Competing interests RS has received honoraria for advisory board participation from MSD, Novartis and QBiotics, and speaking honoraria from BMS. DM has received honoraria for advisory board participation from MSD, BMS and Novartis, and speaking honoraria from BMS and MSD.

Patient and public involvement Patients and/or the public were involved in the design, or conduct, or reporting, or dissemination plans of this research. Refer to the Methods section for further details.

Patient consent for publication Not required.

Provenance and peer review Not commissioned; externally peer reviewed.

Open access This is an open access article distributed in accordance with the Creative Commons Attribution Non Commercial (CC BY-NC 4.0) license, which permits others to distribute, remix, adapt, build upon this work non-commercially, and license their derivative works on different terms, provided the original work is properly cited, appropriate credit is given, any changes made indicated, and the use is non-commercial. See: http://creativecommons.org/licenses/by-nc/4.0/.

ORCID iDs

Jake R Thompson http://orcid.org/0000-0002-0823-3218

Andrea L Smith http://orcid.org/0000-0002-9194-4888

Nadine A Kasparian http://orcid.org/0000-0001-8075-6817

Iris Bartula http://orcid.org/0000-0002-4084-4115

\section{REFERENCES}

1 Carr S, Smith C, Wernberg J. Epidemiology and risk factors of melanoma. Surg Clin North Am 2020;100:1-12.

2 Sung H, Ferlay J, Siegel RL, et al. Global cancer statistics 2020: GLOBOCAN estimates of incidence and mortality worldwide for 36 cancers in 185 countries. CA Cancer J Clin 2021;71:209-49.

3 Ferlay J, Ervik M, Lam F. Global Cancer Observatory: Cancer Today - Melanoma of the skin (C43) fact sheet. Lyon, France: International Agency for Research on Cancer, 2020. Available: https://gco.iarc.fr/ today/fact-sheets-cancers [Accessed 28 May 2021].

4 Australian Institute of Health and Welfare. Cancer data in Australia. cat. No: can 122, 2020. Available: https://www.aihw.gov.au/reports/ cancer/cancer-data-in-australia/contents/summary [Accessed 28 May 2021].

5 Lebel S, Ozakinci G, Humphris G, et al. From normal response to clinical problem: definition and clinical features of fear of cancer recurrence. Support Care Cancer 2016;24:3265-8.

6 McLoone J, Watts K, Menzies S, et al. When the risks are high: psychological adjustment among melanoma survivors at high risk of developing new primary disease. Qual Health Res 2012;22:1102-13.

7 Simard S, Thewes B, Humphris G, et al. Fear of cancer recurrence in adult cancer survivors: a systematic review of quantitative studies. $J$ Cancer Surviv 2013;7:300-22.

8 Tauber NM, O'Toole MS, Dinkel A, et al. Effect of psychological intervention on fear of cancer recurrence: a systematic review and meta-analysis. J Clin Oncol 2019;37:2899-915.

9 Dieng M, Butow PN, Costa DSJ, et al. Psychoeducational intervention to reduce fear of cancer recurrence in people at high risk of developing another primary melanoma: results of a randomized controlled trial. J Clin Oncol 2016;34:4405-14.

10 Dieng M, Kasparian NA, Morton RL, et al. The melanoma care study: protocol of a randomised controlled trial of a psycho-educational intervention for melanoma survivors at high risk of developing new primary disease. BMC Psychol 2015;3:23.

11 Kasparian NA, Mireskandari S, Butow PN, et al. "Melanoma: Questions and Answers." Development and evaluation of a psychoeducational resource for people with a history of melanoma. Support Care Cancer 2016;24:4849-59.

12 Dieng M, Morton RL, Costa DSJ, et al. Benefits of a brief psychological intervention targeting fear of cancer recurrence in people at high risk of developing another melanoma: 12-month follow-up results of a randomized controlled trial. Br J Dermatol 2020;182:860-8.

13 Dieng M, Khanna N, Kasparian NA, et al. Cost-Effectiveness of a Psycho-Educational intervention targeting fear of cancer recurrence in people treated for early-stage melanoma. Appl Health Econ Health Policy 2019;17:669-81.

14 Bauer MS, Damschroder L, Hagedorn H, et al. An introduction to implementation science for the non-specialist. BMC Psychol 2015;3:32

15 Curran GM, Bauer M, Mittman B, et al. Effectiveness-implementation hybrid designs: combining elements of clinical effectiveness and implementation research to enhance public health impact. Med Care 2012;50:217-26.

16 Morton RL, Rychetnik L, McCaffery K, et al. Patients' perspectives of long-term follow-up for localised cutaneous melanoma. Eur J Surg Oncol 2013;39:297-303.

17 Simard S, Savard J. Fear of cancer recurrence inventory: development and initial validation of a multidimensional measure of fear of cancer recurrence. Support Care Cancer 2009;17:241-51.

18 Hingorani AD, Windt DAvander, Riley RD, et al. Prognosis research strategy (progress) 4: stratified medicine research. $B M J$ 2013;346:e5793.

19 Simard S, Savard J. Screening and comorbidity of clinical levels of fear of cancer recurrence. $J$ Cancer Surviv 2015;9:481-91.

20 Fardell JE, Jones G, Smith AB, et al. Exploring the screening capacity of the fear of cancer recurrence Inventory-Short form for clinical levels of fear of cancer recurrence. Psychooncology 2018;27:492-9.

21 Sekhon M, Cartwright M, Francis JJ. Acceptability of healthcare interventions: an overview of reviews and development of a theoretical framework. BMC Health Serv Res 2017;17:88.

22 Keith RE, Crosson JC, O'Malley AS, et al. Using the consolidated framework for implementation research (CFIR) to produce actionable findings: a rapid-cycle evaluation approach to improving implementation. Implement Sci 2017;12:15.

23 Costa DSJ, Dieng M, Cust AE, et al. Psychometric properties of the fear of cancer recurrence inventory: an item response theory approach. Psychooncology 2016;25:832-8.

24 Lovibond SH, Lovibond PF. Manual for the depression anxiety stress scales. 2nd ed. Sydney, NSW: Psychology Foundation of Australia, 1995.

25 Ronk FR, Korman JR, Hooke GR, et al. Assessing clinical significance of treatment outcomes using the DASS-21. Psychol Assess 2013;25:1103-10.

26 Richardson J, Elsworth G, lezzi A. Increasing the sensitivity of the $A Q O L$ inventory for the evaluation of interventions affecting mental health, research paper 61. Centre for Health Economics, Monash University, 2011

27 Weiner BJ, Lewis CC, Stanick C, et al. Psychometric assessment of three newly developed implementation outcome measures. Implement Sci 2017;12:108.

28 Hilsenroth MJ, Blagys MD, Ackerman SJ, et al. Measuring Psychodynamic-Interpersonal and cognitive-behavioral techniques: development of the comparative psychotherapy process scale. Psychol Psychother 2005;42:340-56.

29 Blackburn I-M, James IA, Milne DL, et al. The revised cognitive therapy scale (CTS-R): psychometric properties. Behav Cogn Psychother 2001;29:431-46.

30 Ogrodniczuk JS, Piper WE. Measuring therapist technique in psychodynamic psychotherapies. development and use of a new scale. J Psychother Pract Res 1999;8:142-54.

31 PASS 13 Power Analysis and Sample Size Software [program]. NCSS, LLC. Kaysville, Utah, USA, 2014. Available: www.ncss.com/ software/pass [Accessed 2 Jun 2021].

32 Bell KJL, Mehta Y, Turner RM, et al. Fear of new or recurrent melanoma after treatment for localised melanoma. Psychooncology 2017;26:1784-91.

33 Locascio JJ, Atri A. An overview of longitudinal data analysis methods for neurological research. Dement Geriatr Cogn Dis Extra 2011;1:330-57.

34 Hayes AF, Rockwood NJ. Regression-Based statistical mediation and moderation analysis in clinical research: observations, recommendations, and implementation. Behav Res Ther 2017;98:39-57.

35 Braun V, Clarke V. Using thematic analysis in psychology. Qual Res Psychol 2006;3:77-101.

36 Kan JM, Dieng M, Butow PN, et al. Identifying the 'Active Ingredients' of an Effective Psychological Intervention to Reduce Fear of Cancer Recurrence: A Process Evaluation. Front Psychol. In Press 2021;12.

37 Pinnock H, Barwick M, Carpenter CR, et al. Standards for reporting implementation studies (STARI) statement. BMJ 2017;356:i6795. 
38 Pinnock H, Barwick M, Carpenter CR, et al. Standards for reporting implementation studies (STARI): explanation and elaboration document. BMJ Open 2017;7:e013318

39 International Committee of Medical Journal Editors. Defining the role of authors and contributors, 2021. Available: http://www.icmje.org/ recommendations/browse/roles-and-responsibilities/defining-therole-of-authors-and-contributors.html [Accessed 17 Dec 2021]

40 Lynch FA, Katona L, Jefford M, et al. Feasibility and Acceptability of Fear-Less: A Stepped-Care Program to Manage Fear of Cancer Recurrence in People with Metastatic Melanoma. J Clin Med 2020;9:2969.

41 Butow PN, Bell ML, Smith AB, et al. Conquer fear: protocol of a randomised controlled trial of a psychological intervention to reduce fear of cancer recurrence. BMC Cancer 2013;13:201.

42 Butow PN, Turner J, Gilchrist J, et al. Randomized trial of ConquerFear: a novel, theoretically based psychosocial intervention for fear of cancer recurrence. J Clin Oncol 2017;35:4066-77.

43 Shaw J, Kamphuis H, Sharpe L, et al. Setting an international research agenda for fear of cancer recurrence: an online Delphi consensus study. Front Psychol 2021;12:596682.

44 Butow $P$, Shaw J, Vaccaro L, et al. A research agenda for fear of cancer recurrence: a Delphi study conducted in Australia. Psychooncology 2019;28:989-96.

45 Melanoma Institute of Australia. Your guide to early melanoma (3rd edition), 2021. Available: https://www.melanoma.org.au/ understanding-melanoma/support-patient-stories/patientinformation/patient-guides/ [Accessed 2 Jun 2021].

46 Lebel S, Simard S, Harris C, et al. Empirical validation of the English version of the fear of cancer recurrence inventory. Qual Life Res 2016;25:311-21.

47 Antony MM, Bieling PJ, Cox BJ, et al. Psychometric properties of the 42-item and 21-item versions of the depression anxiety stress scales in clinical groups and a community sample. Psychol Assess 1998;10:176-81.
48 Henry JD, Crawford JR. The short-form version of the depression anxiety stress scales (DASS-21): construct validity and normative data in a large non-clinical sample. Br J Clin Psychol 2005;44:227-39.

49 Fox RS, Lillis TA, Gerhart J, et al. Multiple group confirmatory factor analysis of the DASS-21 depression and anxiety scales: how do they perform in a cancer sample? Psychol Rep 2018;121:10.1177/0033294117727747:548-65.

50 Johnson CE, Bennett KS, Newton J, et al. A pilot study to assess the validity of the DASS-21 subscales in an outpatient oncology population. Psychooncology 2018;27:695-9.

51 Norton PJ, Anxiety D, Scales S. Depression anxiety and stress scales (DASS-21): psychometric analysis across four racial groups. Anxiety Stress Coping 2007;20:253-65.

52 Richardson J, Sinha K, lezzi A. Modelling the utility of health states with the assessment of quality of life $(A Q \circ L) 8 D$ instrument: overview and utility scoring algorithm, research paper 63. Centre for Health Economics, Monash University, 2011.

53 Richardson J, Chen G, lezzi A. Transformations between the assessment of quality of life AQoL instruments and test-retest reliability, research paper 66. Centre for Health Economics, Monash University, 2011.

54 Richardson J, lezzi A. Psychometric validity and the AQoL-8D multi attribute utility instrument, research paper 71 . Centre for Health Economics, Monash University, 2011.

55 Richardson J, Khan M, lezzi A. Preliminary results for the validation of the assessment of quality of life AQoL-8D instrument, research paper 47. Centre for Health Economics, Monash University, 2010.

56 Dieng M, Kasparian NA, Cust AE, et al. Sensitivity of preferencebased quality-of-life measures for economic evaluations in early-stage melanoma. JAMA Dermatol 2018;154:10.1001/ jamadermatol.2017.4701:52-9. 